\title{
PeerLeaders: Herausbildung interpersonaler Kompetenzen in Informatik-Studiengängen durch Peer-Gruppen
}

\author{
Miriam Lohmüller ${ }^{1}$, Manfred Brill ${ }^{2}$ \\ ${ }^{1}$ miriam.lohmueller@hs-kl.de \\ ${ }^{2}$ manfred.brill@hs-kl.de
}

Fachbereich Informatik und Mikrosystemtechnik,

Hochschule Kaiserslautern

DOI: 10.14464/awic.v3i0.269

\begin{abstract}
Interpersonale Kompetenzen sind im IT-Bereich sehr wichtig, da in diesem Sektor viele Teams und Kleingruppen gebildet werden. Das PeerLeaders-Konzept, bei dem höhere Semester Kommilitonen im Basisstudium unterstützen, festigt den Studienerfolg durch gruppendynamische Prozesse und verbessert Verantwortungsübernahme, Empathie-fähigkeit und Networking.

Die Idee basiert darauf, dass Bachelor-Studierende höherer Semester diejenigen aus den ersten Semestern im Basisstudium fördern. Die älteren Studierenden erhalten dafür ECTSPunkte, da das Programm im Rahmen des Seminars „Kommunikations- und Führungstechniken" stattfindet. Die Unterstützung kann sowohl organisatorisch wie fachlich erfolgen. Durch die fachliche Unterstützung setzen sich die höheren Semester erneut mit dem Stoff aus dem Basisstudium auseinander.

Das Coaching der höheren Semester übernehmen Masterstudierende aus dem Seminar „Fortgeschrittene Kommunikation- und Führungstechniken“ mit dem Thema „Teamarbeit“ und erhalten ebenfalls ECTS-Punkte. Da die Peer-Gruppen für jedes Modul neu gebildet werden, ergibt sich automatisch ein Netzwerk zwischen den Studierenden und die Studienzufriedenheit wird erhöht.
\end{abstract}

Keywords: interpersonale Kompetenzen, gruppendynamische Prozesse, Empathiefähigkeit, Networking 


\section{HINTERGRUND}

Das Projekt InfoStuDi (Informatik studieren in der digitalen Gesellschaft) wird vom Stifterverband im Rahmen des Programms Curriculum 4.0 „Auswirkungen der Digitalisierung auf die Gestaltung von Studiengangsreformen an deutschen Hochschulen" gefördert und ist in den Informatik-Studiengängen des Fachbereichs Informatik und Mikrosystemtechnik der Hochschule Kaiserslautern angesiedelt. Im Projekt werden technische Infrastrukturen umgesetzt, die den späteren Arbeitsumgebungen möglichst stark ähneln, und neue Lehr-, Lern- und Prüfungsformen erprobt und evaluiert.

Die Informatik trägt durch den ständigen technologischen Fortschritt maßgeblich zum Digitalisierungsprozess in der Arbeitswelt bei. Diese digitalisierte Arbeitswelt stellt durch wechselnde Arbeitsplätze und Arbeitszeiten sowie hohe Mobilität große Anforderungen an die Anpassungsfähigkeit der Arbeitnehmer (Deleuze, 1993). IT-Projekte werden in Teams und Kleingruppen, häufig zeitlich und räumlich ungebunden, bearbeitet.

Interpersonale Kompetenzen sind gerade für Absolvierende von Informatik-Studiengängen von zentraler Bedeutung und werden vom Arbeitsmarkt als Bestandteil der Hochschulausbildung von Informatikern vorausgesetzt (Berke \& Hettrich, 2017). Zu diesen Kompetenzen gehören: Selbststeuerungskompetenz, Kooperationskompetenz, Lernkompetenz, Empathie und motivationale Kompetenz (Linck, et al., 2013), (Mandl \& Krause, 2001), (Weinert, 2001). Auf diesen Überlegungen basiert die Idee der „PeerLeaders“.

Der Studiengang Angewandte Informatik an der Hochschule Zweibrücken besteht aus einem Bachelor-Studiengang und einem konsekutiven Master-Studiengang. Die MasterStudierenden müssen ab dem Wintersemester 2018/19 Softskills als Pflichtfach belegen.

\section{METHODEN}

Lernen findet immer auch in einem sozialen Umfeld statt. Es ist gleichzeitig Impulsgebung für andere sowie eigene Aneignung von Wissen (Prenzel, 1993). Diese Gleichzeitigkeit muss bei der Gestaltung neuer Lehr- und Lernkonzepte berücksichtigt werden.

Das erfahrungsorientierte Lernen geht von dem Ansatz aus, dass Neues vor allem dann nachhaltig begriffen wird, wenn es an bisherige Erfahrungen sinnvoll anschließt (Arnold \& Siebert, 1995). Erfahrungen sind einerseits Eindrücke, die mit anderen geteilt werden und andererseits Leistungen, die nur einem selbst zur Verfügung stehen. Durch Erfahrungsaustausch wird ein Perspektivwechsel angeregt und neue Handlungsoptionen können entwickelt werden. Der erfahrungsorientierte Ansatz ist somit eher als Prozess der Umstrukturierung vorhandenen Wissens zu sehen und weniger als Neuerwerb von Wissen (Schüßler, 2001).

Diese Erkenntnisse setzen den Rahmen für die folgende Liste für Forderungen an eine Lehrform, die produktives Lernen unterstützt. 
- Erfahrungen und Interessen der Lernenden sollen in die Gestaltung der Lehre einfließen.

- Wissen soll exemplarisch vermittelt werden.

- Die Lernenden sollen Wissen aktiv nutzen können.

- Die Übernahme von Eigenverantwortung bei den Studierenden soll angeregt werden.

- Die Einleitung eines Reflexionsprozesses (Selbstbild/Fremdbild) soll unterstützt werden.

- Sie Studierenden sollen lernen, sich selbst zu organisieren und ihre Zeit zu managen.

- Die Lehrenden sollen in die Lernprozesse einbezogen werden.

\subsection{PEER-GRUPPEN}

Bei unseren Überlegungen haben wir das Leitmotiv der Peer-Guppen gewählt. In einer PeerGruppe haben Jugendliche einen sozialen Freiraum, in dem sie ihre Möglichkeiten ausloten können. Die Gruppe billigt Verhaltensweisen, die außerhalb riskant wären oder unterbunden würden (Kleiber, Appel, \& Pforr, 1998).

„Peer-Groups sind Gruppen von etwa gleichaltrigen Kindern oder Jugendlichen (Jugendgruppen, Cliquen, Banden). „Peer" bedeutet auch gleich sein bezüglich des Rangs und Status, folglich ist das Alter nur ein Kriterium neben dem des Status. "(Stangl, 2018)

Der Name „PeerLeaders“ wurde in Anlehnung an „Cheerleaders“ im American Football, bei dem Team- und Begeisterungsfähigkeit und ständige Weiterentwicklung gefordert sind, gewählt. Der Name soll ausdrücken, dass jeder Projektteilnehmer gleichzeitig der Lehrende und Lernende ist. Beide Gruppen, die Studierenden im Basisstudium sowie die in den älteren Semestern, werden als PeerLeaders bezeichnet, da sich der Name aus „Peers“ und „Leaders“ zusammensetzt. Mit dem gemeinsamen Namen soll das Zusammengehörigkeitsgefühl in der Gruppe gestärkt werden.

Die PeerLeaders sind als Gruppe von ungefähr Gleichaltrigen geplant, in der sich die Studierenden innerhalb der ersten drei Fachsemester der Bachelorstudiengänge orientieren und sich an den älteren und erfahreneren Studierenden ausrichten. Eigene Werte und Verhaltensweisen sowie Selbstorganisation entwickeln die Studierenden durch das angestrebte Networking (Deci \& Ryan, 1993). Gerade die Studierenden im Studienfach Informatik, die als introvertiert gelten (Jaglo, 2013) (Kohler, 2015), erhöhen durch diese soziale Gruppenbildung ihre kommunikativen und empathischen Fähigkeiten.

\subsection{PEER-TEACHING}

Peer-Gruppen eröffnen neue Lernmethoden, die Lernen in außerschulischer Weise und so den Erwerb von sozialen Kompetenzen fördern. 
„(Das Peer-Teaching ist eine) Methode der Lernunterstützung, bei welcher Peers als CoLehrende für Lernende tätig werden. Es bietet die Möglichkeit, Lernenden Selbstregulierung und Kontrolle über ihr eigenes Lernen zu vermitteln, so dass sie ihre eigene Lehrperson werden können." (Hattie, 2013).

Beim Peer-Teaching ist es wichtig, den Stoff so aufzubereiten, dass er gut verstanden werden kann. Es ist kein spontaner Prozess des Sich-gegenseitig-Helfens, sondern ein Konzept mit klaren Vorgaben und Zielen. Die Vorteile des Peer-Teaching sind:

- Die Mitwirkenden erwerben Kompetenzen in den Bereichen Präsentation, Moderation und Teamarbeit.

- Die Behandlung des Stoffs erfolgt intensiver, wenn Kommilitonen inn aufbereiten, da sie sich schon als Lernende mit dem Stoff beschäftigt haben.

- Soziales Lernen wird gefördert.

- Es ist eine Erfahrung, an die man sich besser erinnern kann als an eine Lerneinheit zu Hause (Jenert, 2008).

\section{KONKRETE UMSETZUNG}

Die Grundidee der PeerLeaders ist, dass Studierende aus höheren Bachelor-Semestern bzw. Masterstudierende ihre Kommilitonen im Basisstudium unterstützen. Die Fachprüfungsordnung des konsekutiven Master-Studiengangs Informatik sieht das Modul "Softskills“ als Pflichtfach vor. Analog ist das Modul „Führungs- und Kommunikationstechnik“ ein Pflichtfach für alle vier Bachelor-Studiengänge der Informatik im Fachbereich. Die Studierenden aus höheren Semestern, die am Projekt PeerLeaders teilnehmen, erhalten bei erfolgreicher Teilnahme ECTS-Punkte in ihrem Studiengang. Die Lehre erfolgt somit in drei Schritten, wie Abbildung 1 zeigt. 


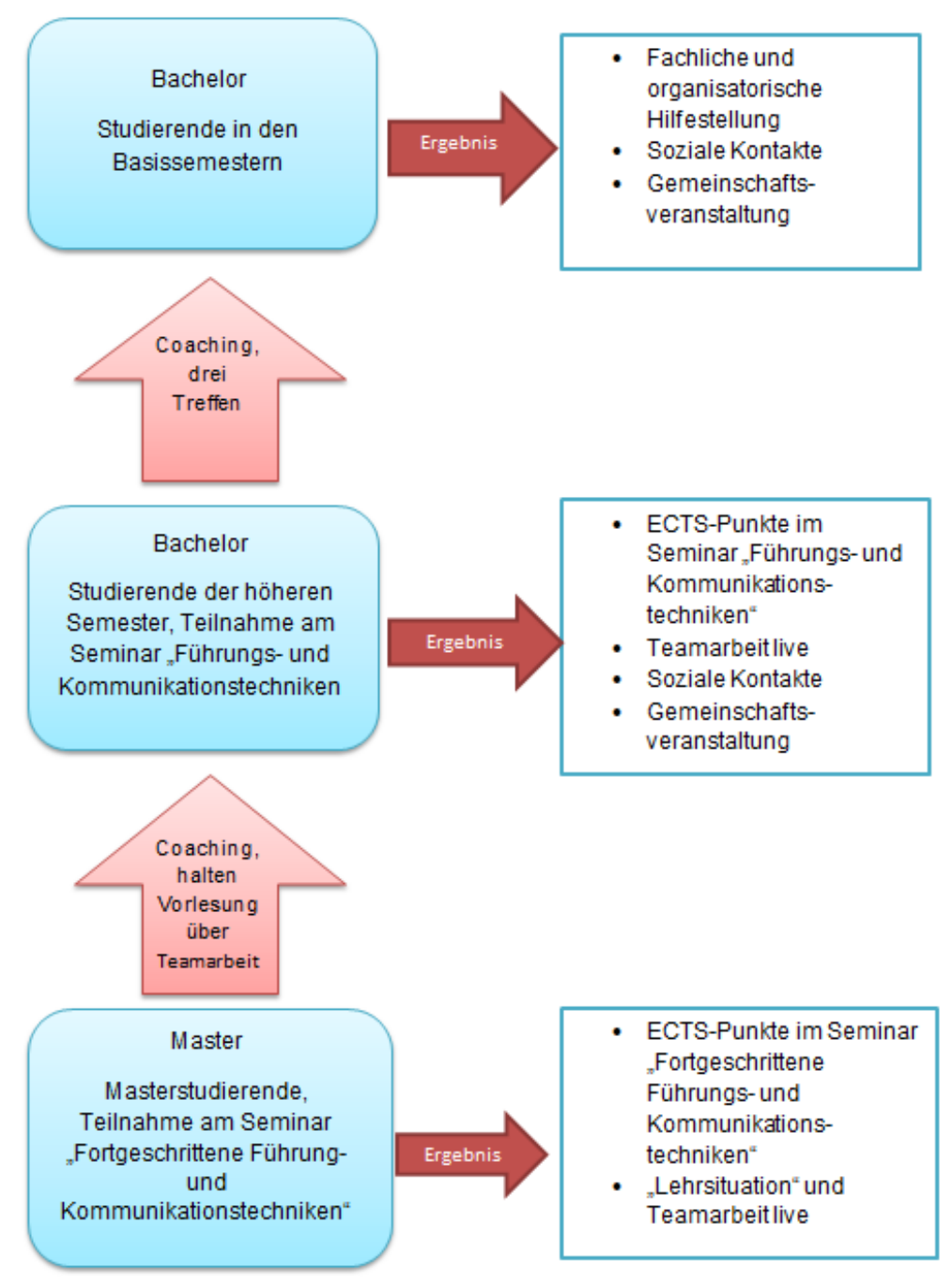

Abbildung 1: Schaubild PeerLeaders

Quelle: $\quad$ Miriam Lohmüller

$\mathrm{Zu}$ Beginn des Vorhabens wurde von einer rein organisatorischen Unterstützung in Studienangelegenheiten seitens der älteren Studierenden ausgegangen. Versuche mit Professoren und wissenschaftlichen Mitarbeitern des Fachbereichs in der Vergangenheit haben jedoch gezeigt, dass auch eine fachliche Unterstützung wichtig ist, wie zum Beispiel das gemeinsame Lösen von Übungsaufgaben aus Lehrveranstaltungen. Das Coaching erfolgt teilweise digital, da die Selbsttests, wie z. B. der Test „Teamrollen nach Belbin Selbsteinschätzung“, mittels der Lernplattform OpenOLAT (OpenOLAT, 2018) durchgeführt werden sollen.

Die Peer-Gruppen werden in jedem Semester neu zusammengestellt, so dass sich unter den Studierenden ein Netzwerk bildet. Dies sorgt für ein Weiterbestehen der sozialen Interaktion 
über den Kurs hinaus. Die Kontaktaufnahme der PeerLeaders untereinander kann neben der studentischen E-Mail auch mit dem Einsatz von sozialen Netzwerken wie Facebook oder WhatsApp oder über OpenOLAT erfolgen und ergänzt so sinnvoll den persönlichen Kontakt.

Als Nachbereitung am Ende des Projekts werden die Projektleiter mit allen Beteiligten eine selbstorganisierte Gemeinschaftsveranstaltung durchführen, um bei den Studierenden das Gefühl, Mitglied einer Gruppe von Vorreitern eines neuen studentischen Networkings zu sein, zu verstärken. Diese Veranstaltung wird sich an einer Retrospektive (Gloger, 2001) in einem agilen Scrum-Projekt orientieren, wie sie in Software-Projekten state-of-the-art ist.

Zur Förderung des Gefühls der Zusammengehörigkeit wurde ein Logo erstellt, das in Abbildung 2 dargestellt ist. Die PeerLeaders erhalten nach Abschluss des Projekts ein Präsent mit diesem Logo. Damit wird angestrebt, die Rolle der PeerLeaders bekannt zu machen und für den nächsten „Durchlauf“ im folgenden Semester zu werben.

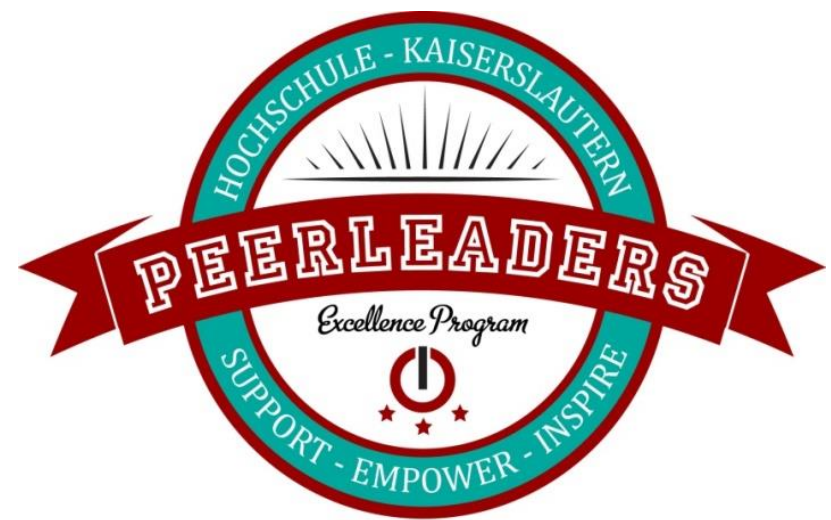

Abbildung 2: Logo Peerleaders

Quelle: $\quad$ Frank Glencairn, www.thepost.de

\section{ERWARTETE ERGEBNISSE}

Lernen soll einen sozialen Charakter haben, Wissen soll gemeinsam mit anderen Lernenden erarbeitet werden und nachhaltig sein. Deshalb formulieren wir die folgenden Thesen:

- Mit dem Konzept der PeerLeaders wird die abstrakte Zukunftsebene mit der Aussicht auf einen Beruf in leitender Position („Wenn sie mal Teamleiter sind ... „) durch eine ähnlich gelagerte Situation ersetzt, in der die Studierenden Themen aus dem Bereich Führung und Kommunikation „live“ erleben können.

- Dieses aktive Wissen zu Führung und interpersoneller Kommunikation trägt zur eigenen Persönlichkeitsentwicklung bei.

- Die Studierenden im Basisstudium erhalten Hilfestellung bei der Bewältigung von organisatorischen und fachlichen Problemen. Dies kann mittelfristig an den Auswirkungen auf die Noten bzw. der Anzahl der ECTS-Punkte gemessen werden. Die Messung der Studienzufriedenheit ist mit einem Online-Fragebogen möglich. 
- Mit der Veränderung der Lernstruktur kann sich auch die Lernkultur der Studierenden verändern. Sie lernen durch die Peer-Gruppen das Arbeiten in Teams und Kleingruppen kennen und können diese Erfahrung im späteren beruflichen Alltag nutzen.

Das Projekt PeerLeaders wird im Sommersemester 2018 erstmals umgesetzt. In den darauf folgenden Semestern wird das Vorhaben ausgeweitet. Bei den Informatik-Studierenden stoßen die PeerLeaders auf sehr großes Interesse. Neben Selbsteinschätzungsfragen werden die folgenden Punkte in der Evaluation qualitativ und quantitativ erfasst und analysiert:

- Mit welchen Medien erfolgt die Kommunikation innerhalb der Studierendengruppe?

- Wie erfolgt die Kommunikation mit den Projektleitern?

- Wie können die PeerLeaders die Gruppenbildung aktiv unterstützen?

- Wie können die Projektleiter die PeerLeaders unterstützen?

- Wie führt man eine solche Kleingruppe in Vorbereitung auf das spätere Arbeitsleben aus Sicht der höheren Semester?

\section{AUSBLICK}

Da die PeerLeaders an der Hochschule sehr große Beachtung erfahren, ist geplant, diese Lehrform auf eine größere Anzahl von Studiengängen in allen Fachbereichen zu übertragen. Ziel ist, die Interaktion zwischen den Studierenden der einzelnen Semester zu steigern und die Zahl der kompetenten Ansprechpartner zu erhöhen. Auf Dauer wird diese Lehrform unserer Ansicht nach die Studienzufriedenheit und den Studienerfolg erhöhen und die Berufsfähigkeit der Studierenden deutlich verbessern.

\section{LITERATURVERZEICHNIS}

Arnold, R., \& Siebert, H. (1995). Konstruktivistische Erwachsenenbildung. Von der Deutung zur Konstruktion von Wirklichkeit. Hohengehren: Schneider.

Berke, Y., \& Hettrich, H. (2017). Marktanalyse im Rahmen der Re-Akkreditierung der Informatik-Studiengänge. Kaiserslautern: Hochschule Kaiserslautern.

Deci, E. L., \& Ryan, R. M. (März 1993). Die Selbstbestimmungsthorie der Motivation und ihre Bedeutung für die Pädagogik. Zeitschrift für Pädagogik 39, Heft 2, S. 223-238.

Deleuze, G. (1993). Unterhandlungen 1972-1990. Suhrkamp.

GI-Empfehlungen. (2016). Empfehlungen für Bachelor- und Masterprogramme im Studienfach Informatik an Hochschulen. Gesellschaft für Informatik e.V.

Glencairn, F. PeerLeader Logo. PeerLeader Logo. Augsburg.

Gloger, B. (2001). Scrum - Produkte zuverlässig und schnell entwickeln. München: Carl Hanser Verlag.

Hattie, J. A. (2013). Lernen sichtbar machen (Überarbeitete deutschsprachige Ausgabe von "Visible Learning" Ausg.). Hohengehren: Schneider.

Jaglo, M. (2013). Hardwarefreaks und Kellerkinder - Klischeevorstellungen über Informatik und die Auseinandersetzung der Studierenden damit. Informatik Spektrum, 36(3), 274-277.

Jenert, T. (2008). Ganzheitliche Reflexion auf dem Weg zum selbstorganisierten Lernen. Bildungsforschung, 5, 1-18. 
Kleiber, D., Appel, E., \& Pforr, P. (1998). Peer Education in der Präventionsarbeit. Entwicklungslinie, Begründungsmuster, Erfahrungen und Entwicklungsanforderungen. Freie Universität Berlin. Berlin: Institut für Prävention und psychosoziale Gesundheitsforschung.

Kohler, K. (Februar 2015). Nerds als Helden - Ein wenig mehr Silicon Valley in Deutschland wäre schön. Informatik-Spektrum, Band 38, Heft 1, S. 37-40.

Linck, B., Ohrndorf, L. S., Stechert, P. M., W., N., Neugebauer, J., \& Schaper, N. (2013). Competence model for informatics modelling and system comprehension. IEEE EDUCON 2013, (S. 85-93). Berlin.

Mandl, H., \& Krause, U.-M. (2001). Lernkompetenz für die Wissensgesellschaft (Forschungsbericht Nr. 145). München: Ludwig-Maximilians-Universität, Lehrstuhl für empirische Pädagogik und Pädagogische Psychologie.

OpenOLAT. (25. Mai 2018). Von OpenOLAT: https://olat.vcrp.de/dmz/ abgerufen

Prenzel, M. (1993). Autonomie und Motivation im Lernen Erwachsener. Zeitschrift für Pädagogik, 239-253.

Schüßler, I. (2001). Frauenbildung als erfahrungsbezogener Ansatz. In W. Gieseke, Handbuch zur Frauenbildung (S. 149-156). Opladen: Leske+Budrich.

Stangl, W. (19.. April 2018). Peergroup. Von Lexikon für Psychologie und Pädagogik: http://lexikon.stangl.eu/161/peergroup/ abgerufen

Weinert, F. E. (2001). Leistungsmessung in Schulen. Weinheim und Basel: Beltz Pädagogik. 